\title{
Hadron cancer therapy complex using nonscaling fixed field alternating gradient accelerator and gantry design
}

\author{
E. Keil* \\ CERN, Geneva, Switzerland \\ A. M. Sessler \\ Lawrence Berkeley National Laboratory, Berkeley, California 94720, USA \\ D. Trbojevic \\ Brookhaven National Laboratory, Upton, New York 11973, USA
}

(Received 7 September 2006; published 29 May 2007)

\begin{abstract}
Nonscaling fixed field alternating gradient (FFAG) rings for cancer hadron therapy offer reduced physical aperture and large dynamic aperture as compared to scaling FFAGs. The variation of tune with energy implies the crossing of resonances during acceleration. Our design avoids intrinsic resonances, although imperfection resonances must be crossed. We consider a system of three nonscaling FFAG rings for cancer therapy with $250 \mathrm{MeV}$ protons and $400 \mathrm{MeV} / u$ carbon ions. Hadrons are accelerated in a common radio frequency quadrupole and linear accelerator, and injected into the FFAG rings at $v / c=$ $0.1294 . \mathrm{H}^{+} / \mathrm{C}^{6+}$ ions are accelerated in the two smaller/larger rings to 31 and $250 \mathrm{MeV} / 68.8$ and $400 \mathrm{MeV} / u$ kinetic energy, respectively. The lattices consist of doublet cells with a straight section for $\mathrm{rf}$ cavities. The gantry with triplet cells accepts the whole required momentum range at fixed field. This unique design uses either high-temperature superconductors or superconducting magnets reducing gantry magnet size and weight. Elements with a variable field at the beginning and at the end set the extracted beam at the correct position for a range of energies.
\end{abstract}

DOI: 10.1103/PhysRevSTAB.10.054701

PACS numbers: 29.20.- c, 29.27.Eg, 41.75.Ak

\section{INTRODUCTION}

Cancer proton therapy exists today in many medical facilities and many more are being built and planned throughout the world. These facilities consist of isochronous cyclotrons, scaling fixed field alternating gradients (FFAGs), and synchrotrons. In this paper we consider nonscaling FFAGs [1]. The advantages of nonscaling FFAGs with respect to synchrotrons are the fixed magnetic field and possibilities of higher repetition rates for spot scanning. With respect to cyclotrons the advantage is very much reduced magnet weight and ease of changing the final energy. Because of the possibility of changing energy and location with each spot (having a repetition rate of about $100 \mathrm{~Hz}$ ), the cancerous tumor can be carefully scanned in three dimensions. The energy variation, and transverse location of a spot, allows that the ion energy deposition inside of the patient, because of the Bragg peak, is precisely at the desired location. The nonscaling FFAG adjusts the final energy by adjusting the number of turns during acceleration. The very strong focusing provides not only smaller aperture but also better orbit control than in cyclotrons, thus leading to lower losses.

We have worked on the subject before [2-6]. This paper is an expansion of [6]. Both [6] and this paper go beyond

*Electronic address: Eberhard.Keil@t-online.de our previous work in presenting a complete complex, thus including a new design of the accelerator and, also, design of the gantry as well as discussion of the transfers from one element to the other. We have changed the rf acceleration from the system with high frequency and fixed frequency described in [6], which jumps harmonic number during the acceleration process, back to a frequency-modulated lowfrequency system.

\section{ACCELERATOR COMPLEX}

The facility consists of three rings shown in Fig. 1. The smallest ring 1 is for protons. The largest ring 3 is for $\mathrm{C}^{6+}$ ions. The middle ring 2 is both for protons and $\mathrm{C}^{6+}$ ions. There, the magnetic rigidities $B \rho$ are equal. Thus, changing particle species does not necessitate changing the magnet excitation. The maximum kinetic energies at extraction are $250 \mathrm{MeV}$ for the protons and $400 \mathrm{MeV} / u$ for the carbon ions, as required by the therapy. Momentum $p$ and magnetic rigidity $B \rho$ change by factors $1.9855,3$, and 2.6095 , respectively, in ring 1 , ring 2 , and ring 3 , as presented in Table I. The momentum offsets $\delta p / p$ are defined with respect to the central or reference energies in each ring. In the first ring for protons, the central kinetic energy is $E_{k}=19.46 \mathrm{MeV}$. In the second ring the central kinetic energy for protons is $E_{k}=118.4 \mathrm{MeV}$ while it is $E_{k}=31.185 \mathrm{MeV} / u$ when the same ring is used for carbon ions. The central kinetic energy in the third ring, used 


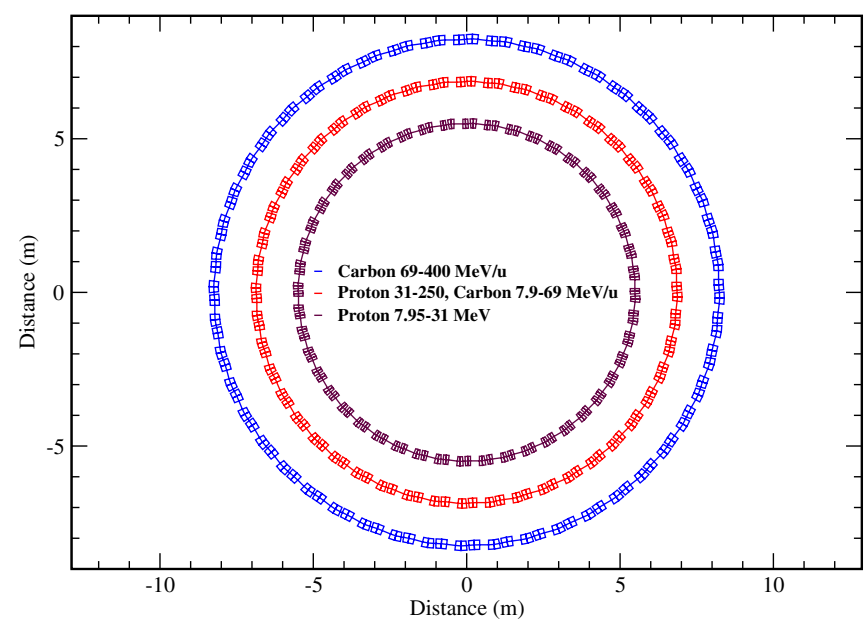

FIG. 1. (Color) Schematic layout of the rings.

only for carbon ions $C^{6+}$, is $E_{k}=208.96 \mathrm{MeV} / u$. Table I also shows the parameters that can be calculated without reference to particular lattices. The parameters in the 1-Inj column of the ring 1 in Table I are chosen such that the particle speed $c \beta$ of the protons at injection into ring 1 and that of the $\mathrm{C}^{6+}$ ions at injection into ring 2 are equal, thus permitting the acceleration of both species in the same system of radio frequency quadrupole and linear accelerator. In all rings, the rf frequencies are proportional to the particle speed $c \beta$. We assume that the $\mathrm{H}^{+}$bunches in ring 1 and the $\mathrm{C}^{6+}$ bunches in ring 2 occupy only every second bucket, and halve the harmonic numbers for $\mathrm{H}^{+}$ions in ring 2 and for $\mathrm{C}^{6+}$ ions in ring 3.

\section{A. Lattices}

Table II shows the lattice parameters in the proton and carbon machines. There is much similarity between them. All have 48 periods. The cell lengths are chosen in the ratio 4:5:6. As will be discussed in Sec. IIC, we propose to accelerate with frequency-modulated rf systems.

For the focusing in all lattices, we have a choice between doublets and triplets of combined function magnets. An argument in favor of doublets is the smaller magnet count in the ratio 2:3. Arguments in favor of triplets are a smaller ratio of the reverse bending in the $F$ magnets to the bending in the D magnet, and, hence, smaller dipole fields, and the symmetry points at the center of the $\mathrm{D}$ magnet and at the center of the straight section between the triplets, where $\alpha_{x}, \alpha_{y}$, and $D_{x}^{\prime}$ vanish. We adopt doublet lattices. The straight section space for the rf cavities, $0.29 \mathrm{~m}$, and the gap between the magnets, $0.08 \mathrm{~m}$, are the same in all lattices. Figures 2-4 show the schematic layout and the orbit functions in ring 1 , ring 2 , and ring 3 , respectively, at their respective reference momenta. The dispersion $D_{x}$ is very small.

The bending angles in the cells are adjusted such that the path length variation at the reference energy with the relative momentum deviation $\delta p / p$ approximately vanishes to first order. The remaining path length variation is predominantly quadratic in $\delta p / p$. This is achieved by bending the beam away from the machine center in the horizontally focusing $\mathrm{F}$ magnets.

A similar design principle had been adopted in FFAG rings for accelerating muons [7] and their electron model

TABLE I. Beam parameters of $\mathrm{H}^{+}$and $\mathrm{C}^{6+}$ in rings 1,2 , and 3. Identical particle parameters at $n$-Extr and $(n+1)$-Inj.

\begin{tabular}{lcccccccr}
\hline \hline \multicolumn{1}{c}{\begin{tabular}{c} 
Particle \\
\multicolumn{1}{c}{ Ring }
\end{tabular}} & \multicolumn{3}{c}{$\mathrm{H}^{+}$} & & \multicolumn{3}{c}{$\mathrm{C}^{6+}$} \\
1-Inj & 1-Extr & 2-Inj & 2-Extr & 2-Inj & 2-Extr & 3-Inj & 3-Extr \\
\hline Kinetic energy $/ u(\mathrm{MeV})$ & 7.951 & 30.97 & 30.97 & 250 & 7.8934 & 68.801 & 68.801 & 400 \\
$\beta$ & 0.1294 & 0.2508 & 0.2508 & 0.6136 & 0.1294 & 0.3645 & 0.3645 & 0.7145 \\
$B \rho(\mathrm{T} \mathrm{m})$ & 0.4083 & 0.8107 & 0.8107 & 2.432 & 0.8107 & 2.432 & 2.432 & 6.3472 \\
$\delta p / p$ & -0.3301 & +0.3301 & -0.5 & +0.5 & -0.5 & +0.5 & -0.4459 & +0.4459 \\
\hline \hline
\end{tabular}

TABLE II. Lattice parameters. All rings have 48 cells. The coefficient $K$ is the gradient divided by the magnetic rigidity.

\begin{tabular}{lccc}
\hline \hline \multicolumn{1}{c}{ Ring } & 1 & 2 & 3 \\
Particle & $\mathrm{H}^{+}$ & $\mathrm{H}^{+}$and $\mathrm{C}^{6+}$ & $\mathrm{C}^{6+}$ \\
\hline Cell length $(\mathrm{m})$ & 0.72 & 0.9 & 1.08 \\
Circumference $(\mathrm{m})$ & 34.56 & 43.20 & 51.84 \\
F magnet length $(\mathrm{m})$ & 0.17 & 0.26 & 0.35 \\
F magnet angle (rad) & -0.0743 & -0.1149 & -0.1139 \\
F magnet $K\left(\mathrm{~m}^{-2}\right)$ & 25.37 & 10.64 & 7.06 \\
D magnet length (m) & 0.18 & 0.27 & 0.36 \\
D magnet angle (rad) & 0.2052 & 0.2458 & 0.2448 \\
D magnet $K\left(\mathrm{~m}^{-2}\right)$ & -23.10 & -9.69 & -6.59 \\
\hline \hline
\end{tabular}




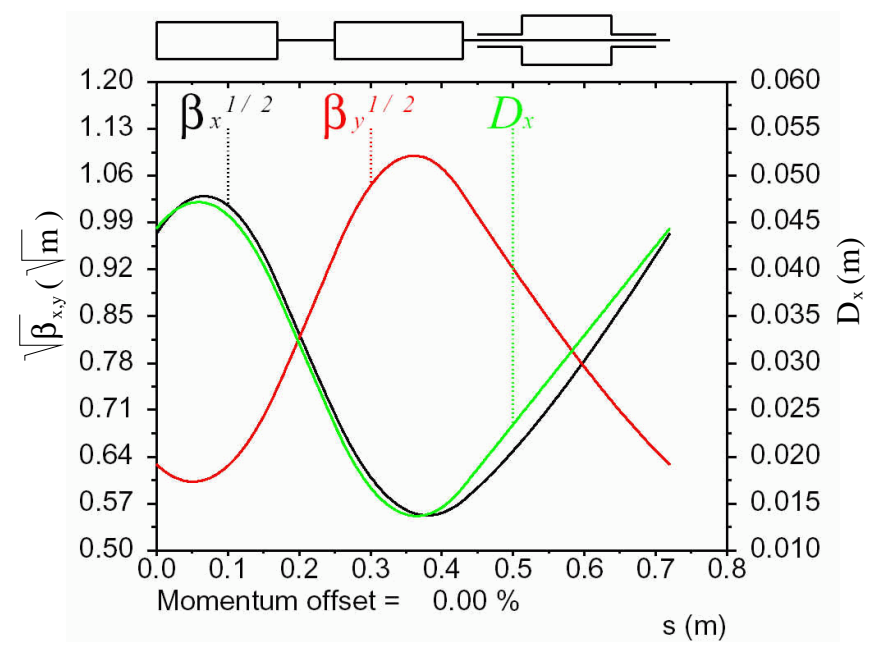

FIG. 2. (Color) Schematic layout and orbit functions in a cell of ring 1 at the reference momentum $p_{\text {ref }}=187.2 \mathrm{MeV} / c$. The left (middle) rectangle is the $F(D)$ magnet. The object at the right is the rf cavity.

[8-10]. Since here both protons and carbon ions are nonrelativistic, the variation of their time of flight with $\delta p / p$ is dominated by the variation of their speed $c \beta$, and the exact path length variation becomes irrelevant.

Adjustable variables are the gradients and the shape of the dipoles. We have chosen the edge angles such that the end faces of the F magnets are parallel, while the angle between the end faces of the $\mathrm{D}$ magnets is twice the bending angle there. The fringe fields are treated in the hard edge approximation. Ring 2 is the most difficult one, since it has the largest ratio of extraction and injection momentum.

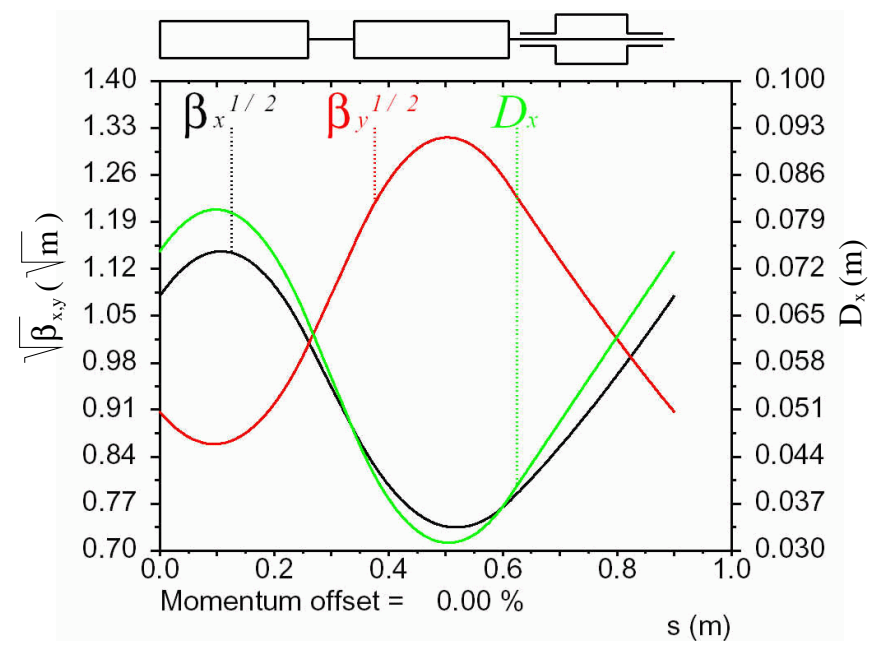

FIG. 3. (Color) Schematic layout and orbit functions in a cell of ring 2 at the proton reference momentum $p_{\text {ref }}=486.1 \mathrm{MeV} / c$ and at the carbon ion reference momentum $p_{\text {ref }}=2.917 \mathrm{GeV} / c$. The left (middle) rectangle is the F (D) magnet. The object at the right is the rf cavity.

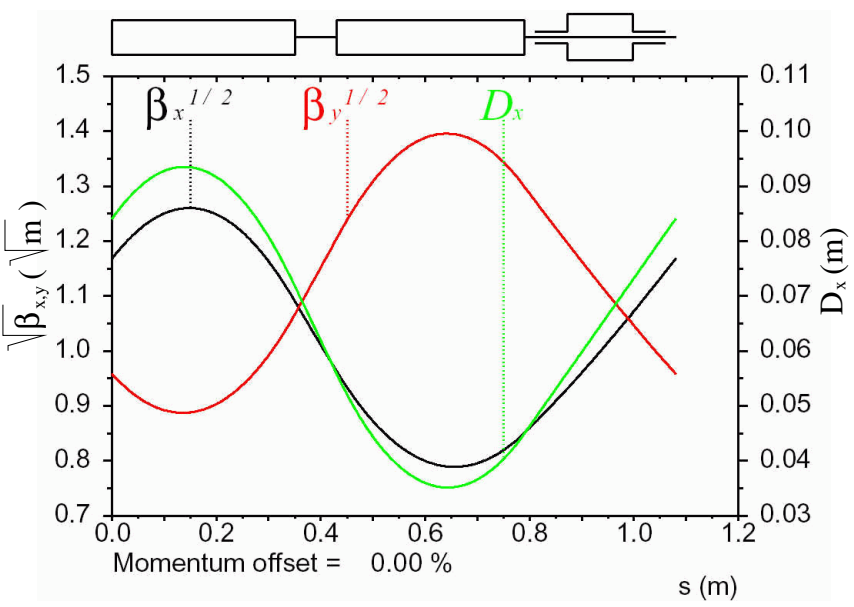

FIG. 4. (Color) Schematic layout and orbit functions in a cell of ring 3 at the reference momentum $p_{\text {ref }}=7.896 \mathrm{GeV} / c$. The left (middle) rectangle is the $F(D)$ magnet. The object at the right is the rf cavity.

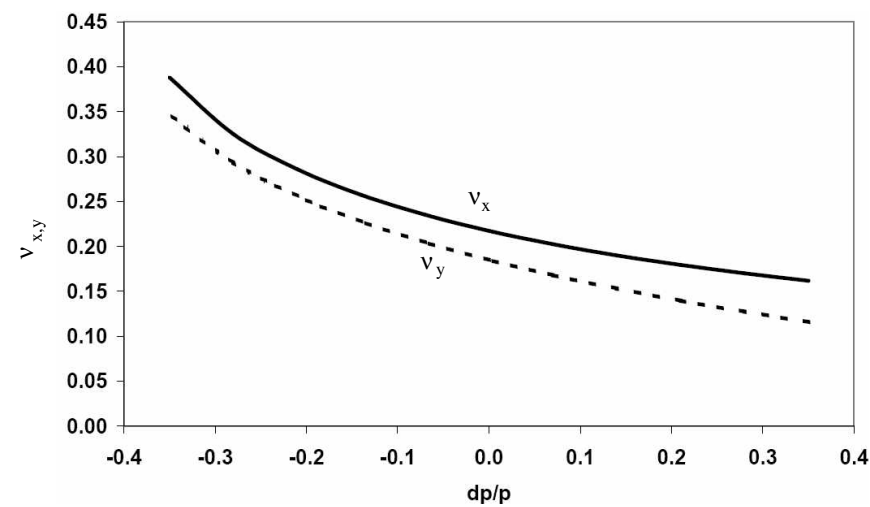

FIG. 5. Horizontal cell tune $\nu_{x}$ and vertical cell tune $\nu_{y}$ in a cell of ring 1 as functions of the relative momentum error $d p / p$ from the reference momentum $p_{\text {ref }}=187.2 \mathrm{MeV} / c$.

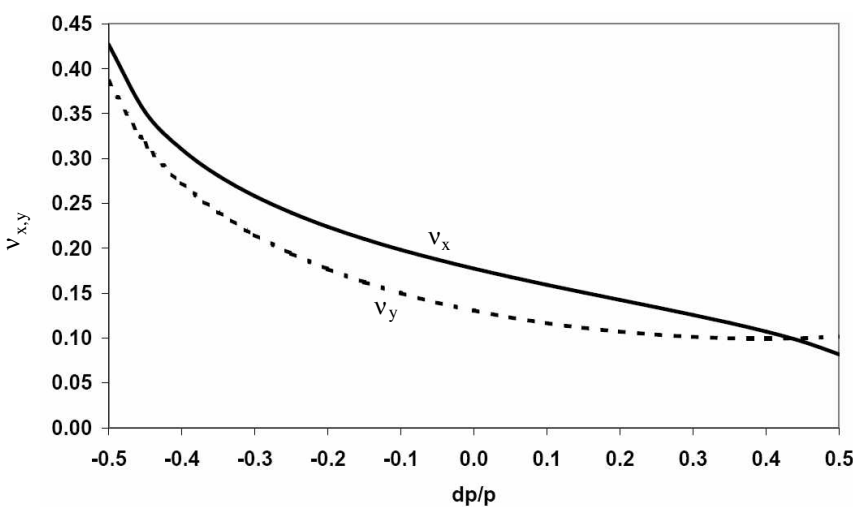

FIG. 6. Horizontal cell tune $\nu_{x}$ and vertical cell tune $\nu_{y}$ in a cell of ring 2 as functions of the relative momentum error $d p / p$ from the proton reference momentum $p_{\text {ref }}=486.1 \mathrm{MeV} / c$ and from the carbon ion reference momentum $p_{\text {ref }}=2.917 \mathrm{GeV} / c$. 


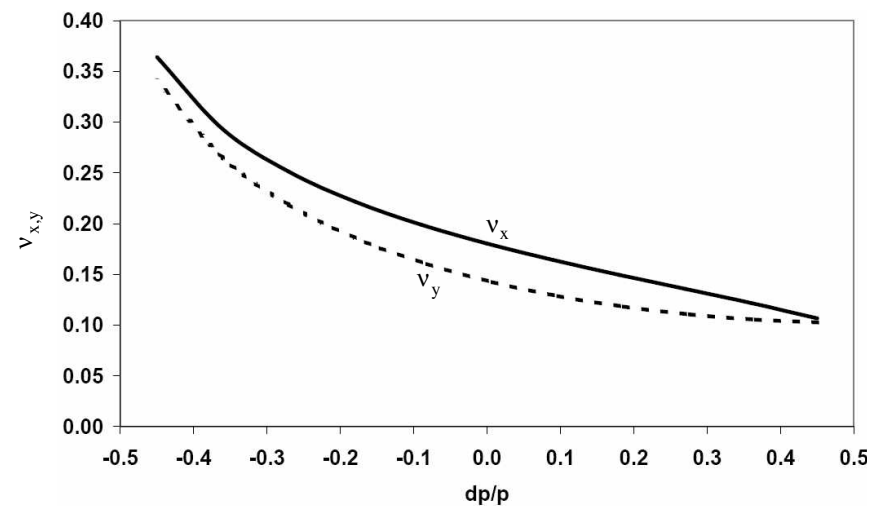

FIG. 7. Horizontal cell tune $\nu_{x}$ and vertical cell tune $\nu_{y}$ in a cell of ring 3 as functions of the relative momentum error $d p / p$ from the reference momentum $p_{\text {ref }}=7.896 \mathrm{GeV} / c$.

The cell tunes $\nu$ at the reference energy are chosen such that both the horizontal $\nu_{x}$ and the vertical $\nu_{y}$ stay below the systematic half integral resonance at $\nu_{x}=\nu_{y}=0.5$ at the lower end and above $\nu_{x}=\nu_{y}=0$ at the upper end of the energy range. Figures 5-7 show the cell tunes $\nu_{x}$ and $\nu_{y}$ in a cell of ring 1 , ring 2 , and ring 3 as a function of the relative momentum deviation $\delta p / p$. In Fig. 5, $\nu_{x}$ and $\nu_{y}$ are well separated, and do not get too close to 0 and 0.5 . In Fig. 6, $\nu_{x}$ and $\nu_{y}$ cross over, and $\nu_{x}$ gets close to 0.5 . Since we assume equal horizontal and vertical emittances throughout, coupling should have no adverse consequences. In Fig. 7, $\nu_{x}$ and $\nu_{y}$ are not as well separated as in Fig. 5, and get closer to 0 and 0.5 than in Fig. 5 .

Figures 8-10 show the maxima of the $\beta$-functions along a cell of ring 1 , ring 2 , and ring 3 , respectively, for the ranges of $\delta p / p$ required according to Table I.

Figures 11-13 show the horizontal orbit offsets $x$ along a cell of ring 1 , ring 2 , and ring 3 , respectively, for several values of $\delta p / p$ in the ranges of $\delta p / p$ required according to Table I. The design involves rapid increase of the

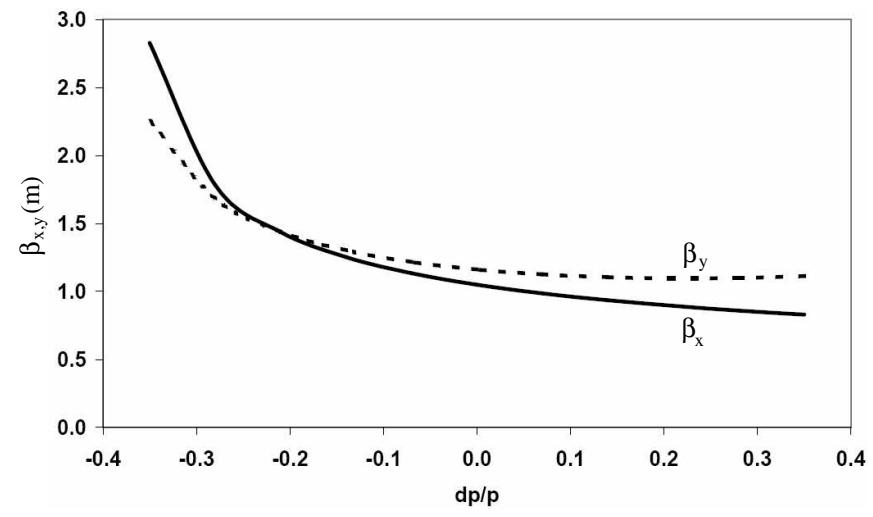

FIG. 8. Maxima of horizontal $\beta$-function $\beta_{x}$ and vertical $\beta$-function $\beta_{y}$ in a cell of ring 1 as functions of the relative momentum error $d p / p$ from the reference momentum $p_{\text {ref }}=$ 187.2 $\mathrm{MeV} / c$.

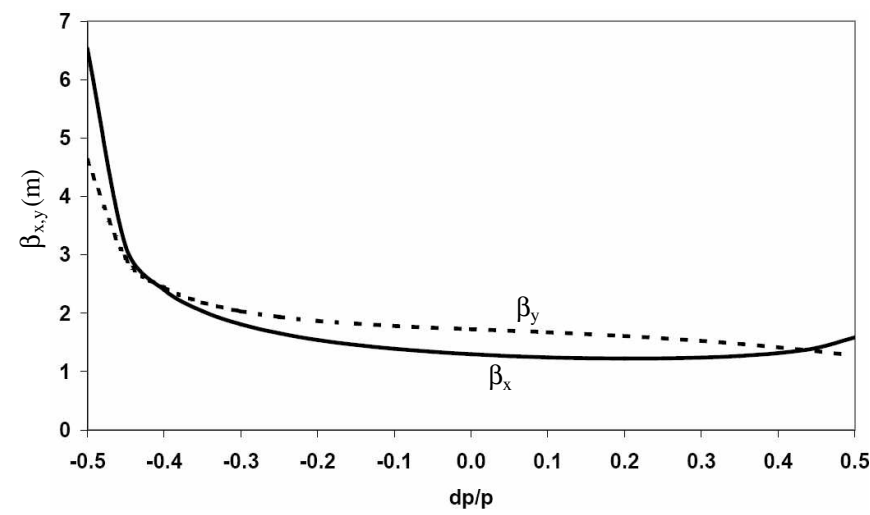

FIG. 9. Maxima of horizontal $\beta$-function $\beta_{x}$ and vertical $\beta$-function $\beta_{y}$ in a cell of ring 2 as functions of the relative momentum error $d p / p$ from the proton reference momentum $p_{\text {ref }}=486.1 \mathrm{MeV} / c$ and from the carbon ion reference momentum $p_{\text {ref }}=2.917 \mathrm{GeV} / c$.

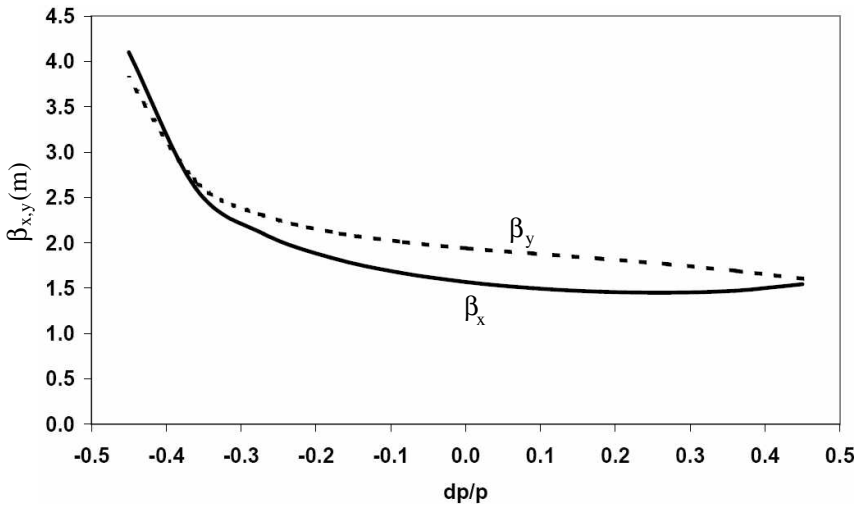

FIG. 10. Maxima of horizontal $\beta$-function $\beta_{x}$ and vertical $\beta$-function $\beta_{y}$ in a cell of ring 3 as functions of the relative momentum error $d p / p$ from the reference momentum $p_{\text {ref }}=$ $7.896 \mathrm{GeV} / c$.

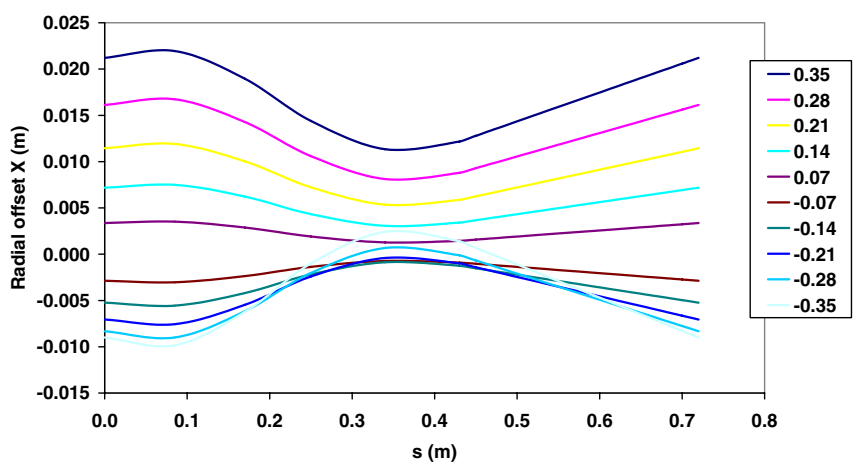

FIG. 11. (Color) Horizontal orbit offset $X$ in $\mathrm{m}$ along a cell of ring 1 for a range of momentum deviation $-0.35 \leq \delta p / p \leq$ +0.35 . 


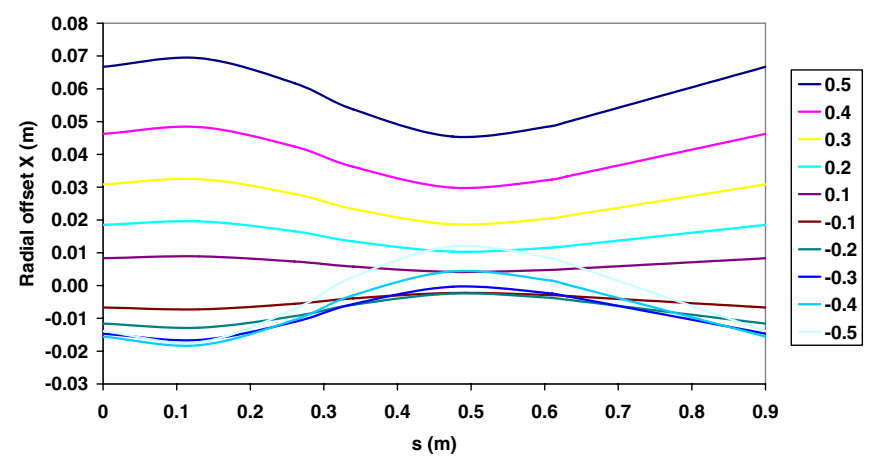

FIG. 12. (Color) Horizontal orbit offset $X$ in $\mathrm{m}$ along a cell of ring 2 for a range of momentum deviation $-0.5 \leq \delta p / p \leq$ +0.5 .

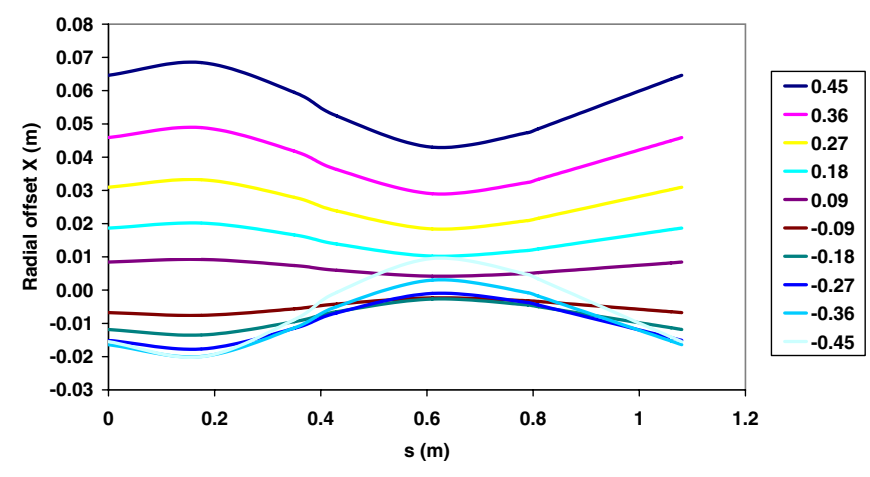

FIG. 13. (Color) Horizontal orbit offset $X$ in $\mathrm{m}$ along a cell of ring 3 for a range of momentum deviation $-0.45 \leq \delta p / p \leq$ +0.45 .

$\beta$-functions at the lower end of the $\delta p / p$ range shown in Figs. $8-10$, and the rapid increase of the radial offset at the upper end shown in Figs. 11-13, but the values are kept within tolerable ranges. The results in Figs. 5-16 were all calculated with the PTC_TWISS command in MAD-X [11].

\section{B. Aperture}

For the calculation of the apertures, we assume a normalized emittance $\varepsilon_{n}=0.5 \times 10^{-6} \pi \mathrm{m}$ in both planes, following [12,13], and allow for five rms beam radii in the half apertures. The vertical aperture radius $A_{y}$ is determined by the amplitude of the vertical betatron oscillations. We use the ranges of $\delta p / p$ from Table I, and take $|\delta p / p| \leq 0.35$ in ring $1,|\delta p / p| \leq 0.5$ in ring 2 , and $|\delta p / p| \leq 0.45$ in ring 3. At all distances $s$ along a cell, we look for the maximum value of $\beta_{y}$ in those ranges, and obtain the vertical aperture $A_{y}$ there. We calculate the contribution of the horizontal betatron oscillations to the horizontal aperture $A_{x}$ in a similar manner. Because of the change of focusing with $\delta p / p$, the radial offset of the closed orbit is asymmetrical with respect to the reference orbit, as can be seen in Figs. 11-13. Hence, we calculate and show the maximum positive and maximum negative horizontal orbit offsets $x$ along a cell in the same ranges of $\delta p / p$. In the F magnets, where the offsets $x$ are largest, the trajectories of the protons with increasing $\delta p / p$ are progressively more to the outside of the reference orbit. In the D magnets, where the offsets $x$ are smallest, the trajectories of the particles with increasing $\delta p / p$ are not in the same order. The trajectory with the smallest $\delta p / p$ is more outside than trajectories with intermediate values of $\delta p / p$.

At all $s$, we subtract 5 times the horizontal $\mathrm{rms}$ betatron beam radius from the maximum negative offset $x$ to find the minimum inner horizontal aperture radius $A_{x}$. Similarly, we add 5 times the horizontal rms betatron beam radius to the maximum positive offset $x$ to find maximum positive horizontal aperture radius $A_{x}$. Figures 14-16 show the maximum negative horizontal aperture radius and maximum positive horizontal aperture radius $A_{x}$ and the vertical aperture radius $A_{y}$ along $s$ in ring 1 , ring 2 , and ring 3 . The latter has the largest aperture of the three rings, since the cells are the longest and the range of $\delta p / p$ is the largest.

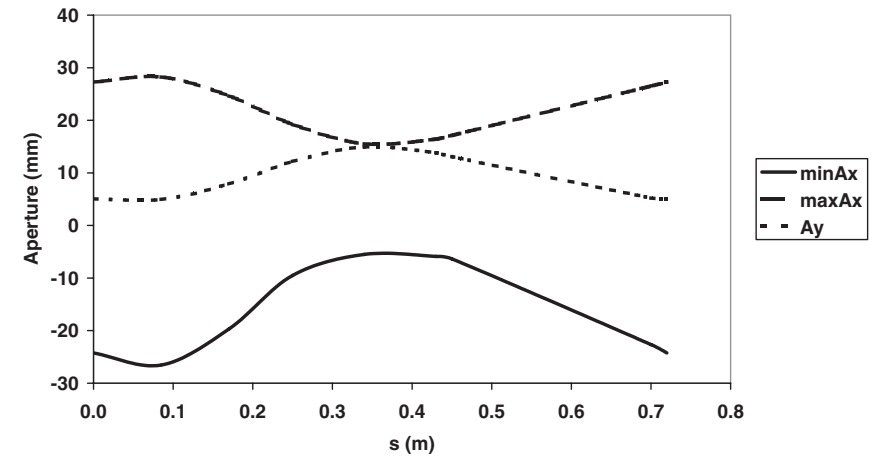

FIG. 14. Maximum negative $\min A_{x}$ and maximum positive $\max A_{x}$ values of the horizontal aperture and vertical half aperture $A_{y}$ in $\mathrm{mm}$ for a cell of ring 1 for $-0.35 \leq \delta p / p \leq+0.35$.

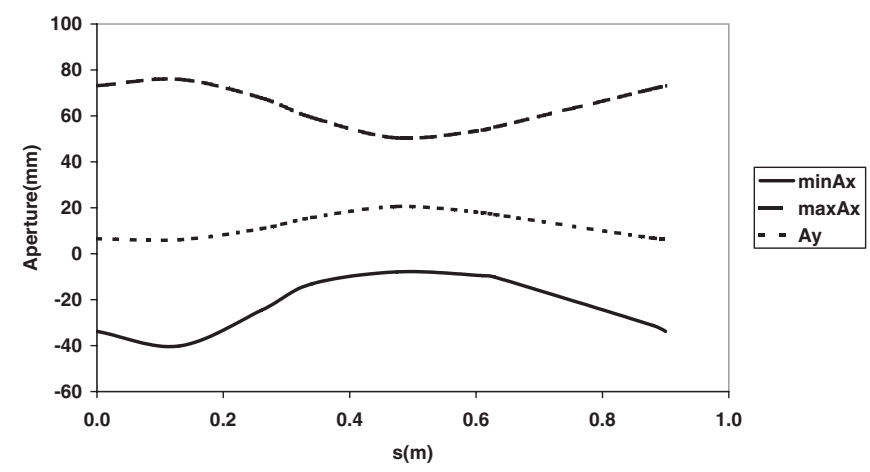

FIG. 15. Maximum negative $\min A_{x}$ and maximum positive $\max A_{x}$ values of the horizontal aperture and vertical half aperture $A_{y}$ in $\mathrm{mm}$ for a cell of ring 2 for $-0.5 \leq \delta p / p \leq+0.5$. 


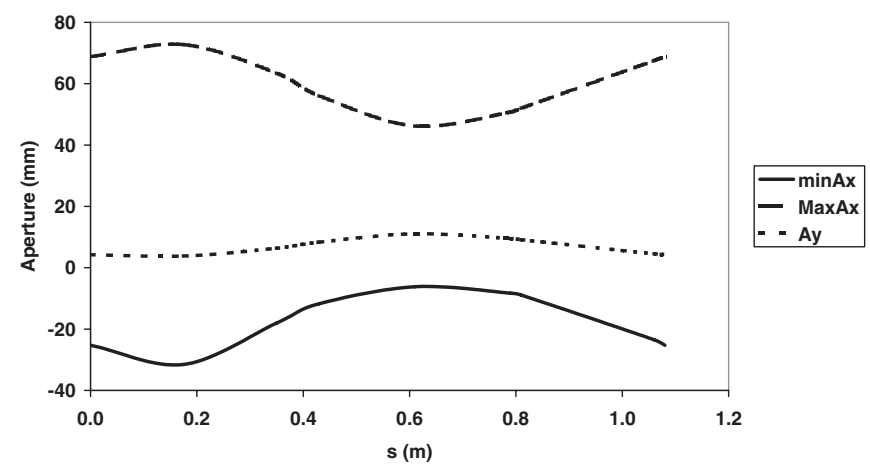

FIG. 16. Maximum negative $\min A_{x}$ and maximum positive $\max A_{x}$ values of the horizontal aperture and vertical half aperture $A_{y}$ in $\mathrm{mm}$ for a cell of ring 3 for $-0.45 \leq \delta p / p \leq+0.45$.

\section{Acceleration}

We adopt frequency-modulated rf systems. In each ring, the harmonic number $h$ is constant, and the rf frequency increases in proportion to the particle speed $c \beta$. The range of frequencies has a lower limit due to the requirement that the harmonic numbers $h$ are in the ratio of circumferences to allow transfer of the bunches in one ring into buckets in the next one. This implies $h=4,5,6$, and minimum frequencies between about 4.2 and $8.7 \mathrm{MHz}$, respectively, in the three rings. The properties of ferrites yield an upper limit for the range of frequencies at about $50 \mathrm{MHz}$. The rf system of the FNAL booster operates at up to $53 \mathrm{MHz}$ [14]. We reduce the range of frequencies in the three rf systems by filling only every second bucket with $\mathrm{H}^{+}$in ring 1 , and with $\mathrm{C}^{6+}$ in ring 2 , and by halving the harmonic numbers for $\mathrm{H}^{+}$in ring 2 and for $\mathrm{C}^{6+}$ in ring 3, and filling neighboring buckets with bunches there. Thus, all frequencies are in the range from about 8.7 to $25.3 \mathrm{MHz}$, as shown in Table III, and the rf systems could be largely the same in all three rings. We have ignored the small variation of the path length with $\delta p / p$ when calculating the frequencies.

The maximum rate of relative frequency change $(\mathrm{d} f / \mathrm{d} t) / f_{i}$, the energy gain in a turn $\Delta E$, the number of turns $N$, the acceleration time $T$, and hence the repetition rate, rf system parameters bucket height $\Delta p / p$, bunch area $A$ and synchrotron tune $Q_{s}$, ring parameters such as circumference $C$, and beam parameters such as rest mass $E_{0}$, and the relativistic parameters $\beta$ and $\gamma$ are all related. We evaluate $(\mathrm{d} f / \mathrm{d} t) / f_{i}$ at injection, where it is largest:

$$
\frac{1}{f_{i}} \frac{\mathrm{d} f}{\mathrm{~d} t}=\frac{c \Delta E}{C E_{0} \beta_{i} \gamma_{i}^{3}} .
$$

Here, $\beta_{i}$ and $\gamma_{i}$ are the values of the relativistic parameters at injection. Clearly, $(\mathrm{d} f / \mathrm{d} t) / f_{i}$ determines $\Delta E$, since all other parameters are already fixed. The number of turns $N$ follows from $N=\left(E_{f}-E_{i}\right) / \Delta E$, and the acceleration time $T$ follows from $N$ by integration:

$$
T=\int_{0}^{N C} \frac{\mathrm{d} s}{c \beta(s)} .
$$

Assuming that the energy change per unit distance $\mathrm{d} E / \mathrm{d} s=\Delta E / C$ is constant, so that the rf system increases the beam energy by the same amount on every turn, the integral becomes in closed form

$$
T=\frac{C N\left(\beta_{f} \gamma_{f}-\beta_{i} \gamma_{i}\right)}{c\left(\gamma_{f}-\gamma_{i}\right)} .
$$

In our design procedure, we fix the repetition frequency, about $1 / 2 T$, and get $\Delta E, N, T$, and also $(\mathrm{d} f / \mathrm{d} t) / f_{i}$. The energy gain per turn $\Delta E$ determines also the rate at which the resonances are crossed.

We envisage rf systems with cavities loaded with modern soft magnetic material, such that they "resonate" in the frequency range needed, and have a high enough permeability for the cavities to have a reasonable size. We adjust their number such that the losses are tolerable.

The bunches are arranged in a train. There are at most 4 $\mathrm{H}^{+}$bunches in rings 1 and 2 , and at most $5 \mathrm{C}^{6+}$ bunches in rings 2 and 3 . Shorter bunch trains may be useful to increase the rise time of the kicker magnets.

An alternative is operating all rings with just one bunch, and to use harmonic numbers $h=2$ in ring $1, h=1$ for $\mathrm{H}^{+}$and $h=2$ for $\mathrm{C}^{6+}$ in ring 2 , and $h=1$ in ring 3. The frequencies would be between 1.8 and $5.1 \mathrm{MHz}$, and in the range where such cavities have already been operated [15].

\section{Magnets}

Table IV shows the apertures and the fields of the combined function magnets. Their shape is discussed in Sec. II A. The inner and outer values of the horizontal aperture radius in the $\mathrm{F}$ and $\mathrm{D}$ magnets are calculated in the manner discussed in Sec. II B. The gradients $G$ in the combined function dipoles cause a variation of the mag-

TABLE III. New rf system parameters, harmonic numbers $h$, initial and final frequencies $f_{i}$ and $f_{f}$, number of turns $N$, acceleration time $T$, initial relative rate of frequency change $(\mathrm{d} f / \mathrm{d} t) / f_{i}$, energy gain per turn $\Delta E$. Also given are bucket height $\Delta p / p$, bucket area $A$, synchrotron tune $Q_{s}$ at the extraction energy, assuming a stable phase angle $\pi / 6$ from the nearest zero crossing.

\begin{tabular}{cccccccccccc}
\hline \hline Ring & Ion & $h$ & $f_{i}(\mathrm{MHz})$ & $f_{f}(\mathrm{MHz})$ & $N$ & $T(\mathrm{~ms})$ & $\frac{\mathrm{d} f / \mathrm{d} t}{f_{i}}(1 / \mathrm{ms})$ & $\Delta E(\mathrm{keV} / u)$ & $10^{3} \Delta p / p$ & $A(\mathrm{meV} \mathrm{s})$ & $10^{3} Q_{s}$ \\
\hline 1 & $\mathrm{H}^{+}$ & 8 & 8.977 & 17.40 & 1500 & 0.9063 & 1.061 & 15.23 & 3.800 & 9.644 & 5.720 \\
2 & $\mathrm{H}^{+}$ & 5 & 8.701 & 21.29 & 3000 & 0.9594 & 1.939 & 72.48 & 5.110 & 77.81 & 6.749 \\
2 & $\mathrm{C}^{6+}$ & 10 & 8.977 & 25.29 & 1500 & 0.8625 & 2.280 & 40.61 & 4.119 & 188.3 & 9.374 \\
3 & $\mathrm{C}^{6+}$ & 6 & 12.65 & 24.79 & 3000 & 0.9192 & 1.519 & 110.4 & 5.100 & 1217 & 7.887 \\
\hline \hline
\end{tabular}


TABLE IV. Magnet parameters, aperture in mm, field $B$ in T.

\begin{tabular}{lcccccc}
\hline \hline \multicolumn{1}{c}{ Magnet } & \multicolumn{1}{c}{$\mathrm{F}$} & & \multicolumn{1}{c}{$\mathrm{D}$} & 3 \\
\hline \multicolumn{1}{c}{ Ring } & 1 & 2 & 3 & 1 & 2 & -12 \\
Inner horizontal aperture radius & -27 & -40 & -32 & -10 & -13 & 56 \\
Outer horizontal aperture radius & 28 & 76 & 73 & 19 & 59 & 3.34 \\
$B$ at inner aperture radius & -0.68 & -1.40 & -2.40 & 0.82 & 1.69 & 1.30 \\
$B$ at outer aperture radius & 0.17 & 0.57 & 0.81 & 0.44 & 0.50 & 11 \\
Vertical half aperture & 8 & 13 & 7 & 15 & 21 & \\
\hline \hline
\end{tabular}

netic field $B$ across the horizontal aperture, quantified in the rows labeled " $B$ at inner/outer aperture radius." The fields in the F magnets change sign inside the horizontal aperture, those in the $\mathrm{D}$ magnets do not.

In ring 1, the fields are below $1 \mathrm{~T}$. The maximum field in ring $2,1.3 \mathrm{~T}$, occurs in the $\mathrm{D}$ magnets at the inner horizontal aperture radius. The magnets in these rings are conventional iron-dominated magnets. They may be excited either by resistive room-temperature coils, or by coils made of high-temperature superconductor [16]. The maximum field in ring 3 also occurs in the D magnets at the minimum horizontal aperture. At about $3.3 \mathrm{~T}$, it is in the range of easy superconducting magnets. Suitable combined function magnets can be arrived at by scaling from the superconducting magnets in the proposed proton transport line for the J-PARC neutrino experiment [17] with $B=2.59 \mathrm{~T}, G=18.7 \mathrm{~T} / \mathrm{m}$, and a coil aperture radius $A=86.7 \mathrm{~mm}$.

\section{E. Injection and extraction}

Extraction happens in two stages: (i) A full-aperture fast kicker magnet in a long straight section deflects the extracted beam such that it is outside the circulating beam in horizontal phase space. The energy of the extracted proton beam can be varied in steps at most equal to the energy gain $\Delta E$ in Table III by accelerating for a variable number of turns, or half that value (in $\mathrm{MeV} / u$ ) for the $\mathrm{C}^{6+}$ beam. We operate the rings with up to $4 \mathrm{H}^{+}$and up to $5 \mathrm{C}^{6+}$ bunches. Because of the circumference ratio, there is a gap of at least two rf cycles for the extraction kicker rise time in ring 2 for $\mathrm{H}^{+}$and in ring 3 for $\mathrm{C}^{6+}$. There, the extraction kickers have the most demanding parameters. The kicker parame-

TABLE V. Extraction kicker parameters.

\begin{tabular}{lccc}
\hline \hline \multicolumn{1}{c}{ Ring } & 1 & 2 & 3 \\
\hline Kick angle (mrad) & 11.5 & 7.6 & 4.5 \\
Rise time (ns) & 120 & 80 & 80 \\
Aperture width (mm) & 52 & 107 & 94 \\
Aperture height (mm) & 28 & 36 & 19 \\
Kicker length (m) & 0.2 & 0.2 & 0.2 \\
Kicker voltage (kV) & 4.0 & 24.7 & 33.4 \\
Kicker field (T) & 0.047 & 0.092 & 0.14 \\
\hline \hline
\end{tabular}

ters are shown in Table V. The horizontal and vertical apertures in the kicker are almost as large as in the F and D magnets, respectively, as expected. All injection kickers have longer rise times. (ii) A septum magnet deflects the extracted beam further, such that it misses components downstream, and sends it into a transfer line. Injection uses similar components in reverse order.

\section{GANTRY}

The beam is assumed to have equal emittances $\varepsilon_{x}=\varepsilon_{y}$. In the fixed transport line from the accelerators to the rotating gantry, it is matched to equal $\beta$-functions, $\alpha_{x}=$ $\alpha_{y}=0$, and $D_{x}=D_{x}^{\prime}=0$ at the junction. A 0.3 -m-long straight matching insertion consists of two quadrupoles and two drift spaces, and rotates with the gantry, but remains on axis. It matches the round beam at the end of the transfer line to the gantry cells at any rotation angle. The gantry cells consist of nonscaling FFAG cells of combined function dipoles, arranged in the order focusing-defocusing-defocusing-focusing. The bending angle of a cell is $\pm \pi / 18$. The bending angles of the $\mathrm{F}$ and D magnets are adjusted such that $D_{x}=D_{x}^{\prime}=0$ at the center of the $\mathrm{D}$ magnet. There, the curvature can be changed without harm to the matching of $\beta$-functions and dispersion $D_{x}$ at $\Delta p / p=0$. At the reference energy

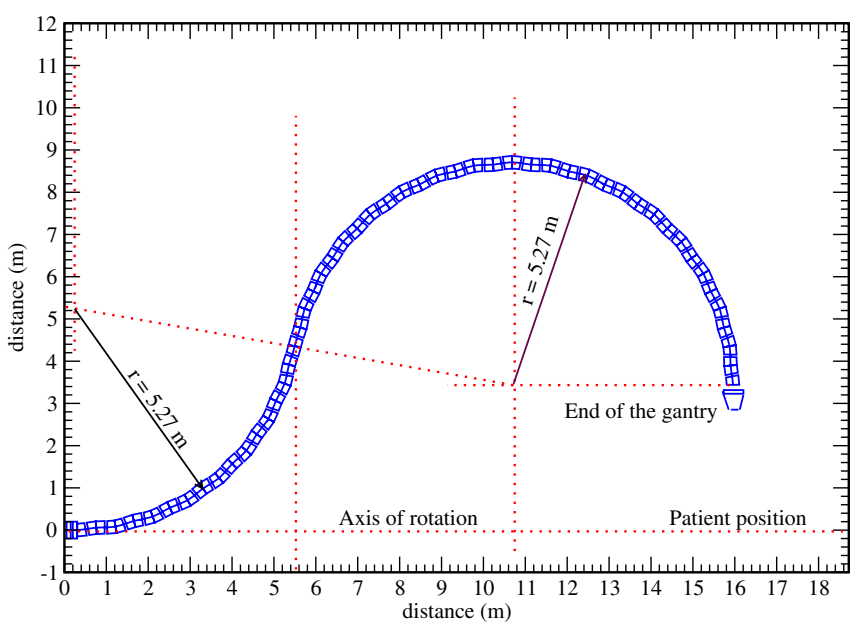

FIG. 17. (Color) Schematic layout of the gantry. 


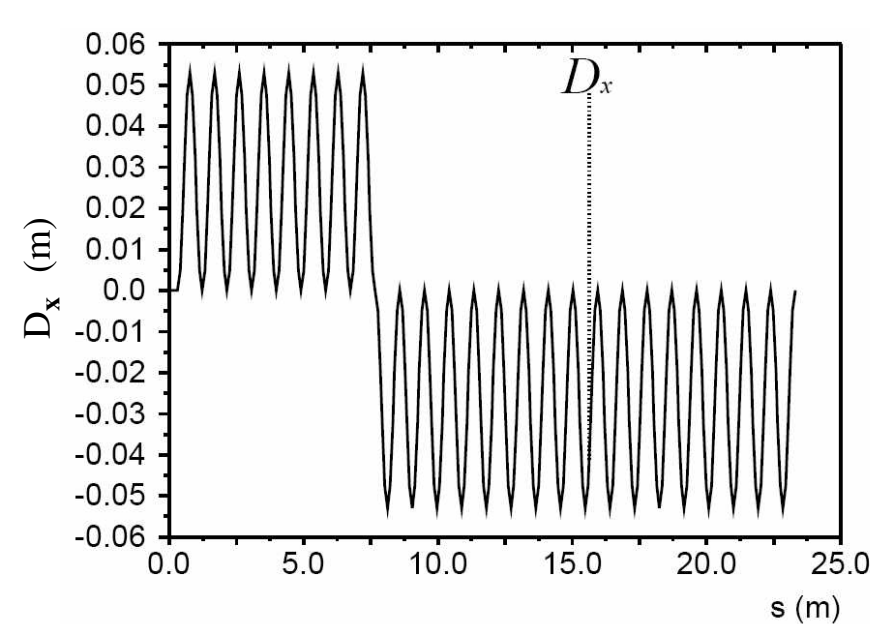

FIG. 18. Horizontal dispersion $D_{x}$ in the gantry.

with $\delta p / p=0$, the maximum $\beta$-functions are less than $1.63 \mathrm{~m}$. The dispersion varies in the range $-55 \leq D_{x} \leq$ $55 \mathrm{~mm}$. Figure 17 shows a schematic layout. Figure 18 shows the matched dispersion. The first eight cells bend the beam away from the axis of rotation. The remaining 17 cells have the opposite curvature, and bend the beam back towards the axis of rotation. The distance between the end of the gantry and the axis of rotation, as well as the maximum distance between the gantry and the axis of rotation, is $9 \mathrm{~m}$ in Fig. 17, and can be varied by changing the number of cells. A similar gantry is in $[18,19]$. With two different magnet excitations, the gantry can transport both $\mathrm{C}^{6+}$ and $\mathrm{H}^{+}$ions. With one excitation it transports $\mathrm{C}^{6+}$ ions with kinetic energies between 150 and $400 \mathrm{MeV} / u$. With about $38 \%$ of that excitation it transports $\mathrm{H}^{+}$with kinetic energies between 90 and $250 \mathrm{MeV}$. A system of deflecting and focusing magnets at the end of the gantry compensates for lateral beam offsets (which are small), allows scanning, and achieves the desired beam size.

\section{CONCLUSIONS}

In this paper we have exhibited a design of three nonscaling FFAGs and a fixed field gantry. We have designed a series of rings producing $200 \mathrm{MeV}$ protons or $400 \mathrm{MeV} / u$ carbon, designed an acceleration scheme, considered injection and extraction, developed a matched beam into the gantry, and presented a gantry design with compact magnets. The complex facility design is now ready for detailed engineering and costing.

\section{ACKNOWLEDGMENTS}

The work of A.M. Sessler was supported by the U.S. Department of Energy under Contract No. DE-AC0205CH11231. D. Trbojevic was supported by the U.S. Department of Energy under Contract No. DE-AC0298CH10886.

[1] C. Johnstone, W. Wan, and A. Garren, Proceedings of the 1999 Particle Accelerator Conference, New York, USA, 1999, p. 3068.

[2] D. Trbojevic, A. G. Ruggiero, E. Keil, N. Neskovic, and A. Sessler, Proceedings of Cyclotrons 2004, Tokyo, Japan, 2004, p. 246, http://ribfweb1.riken.go.jp/cyc2004/ proceedings/data/CYC2004_papers/19P31.pdf.

[3] E. Keil, A. M. Sessler, and D. Trbojevic, at the FFAG04 Workshop, Tsukuba, Japan, 2004, http://hadron.kek.jp/ FFAG/FFAG04_HP/pdf/keil.pdf.

[4] E. Keil, A. M. Sessler, and D. Trbojevic, Proceedings of the 2005 Particle Accelerator Conference, Knoxville, USA, 2005, p. 1667.

[5] D. Trbojevic, E. Keil, and A. Sessler, at the International Symposium on Utilisation of Accelerators, 2005, Dubrovnik, Croatia.

[6] E. Keil, A. M. Sessler, and D. Trbojevic, Proceedings of the 2006 European Particle Accelerator Conference, Edinburgh, 2006, p. 1681.

[7] E. Keil and A. M. Sessler, Nucl. Instrum. Methods Phys. Res., Sect. A 538, 159 (2005).

[8] E. Keil and A. M. Sessler, CERN-AB-2003-095(ABP), 2003.

[9] E. Keil, AB-Note-2004-011 (ABP), 2004.

[10] E. Keil, J. S. Berg, and A. M. Sessler, Proceedings of the 2004 European Particle Accelerator Conference, Lucerne, Switzerland, 2004, p. 587.

[11] http://frs.home.cern.ch/frs/Xdoc/mad-X.html

[12] CERN-2006-006, edited by F. Gerigk et al., 2006.

[13] S. Gammino, http://www.Ins.infn.it/info/LNS ActivityReport_2005/SEZ_E_Ion \% 20Sources \% 20and \% 20Accelerators/E_10.pdf

[14] Q. A. Kerns et al., IEEE Trans. Nucl. Sci. 26, 4111 (1979).

[15] R. Garoby et al., Proceedings of the 2005 Particle Accelerator Conference, Knoxville, USA, 2005, p. 1619.

[16] R. Gupta et al., Proceedings of the 2005 Particle Accelerator Conference, Knoxville, USA, 2005, p. 3016.

[17] T. Nakamoto et al., Proceedings of the 2005 Particle Accelerator Conference, Knoxville, USA, 2005, p. 495.

[18] D. Trbojevic et al., Proceedings of the 2006 European Particle Accelerator Conference, Edinburgh, 2006, p. 352.

[19] D. Trbojevic et al. (unpublished). 